\title{
Empirical distribution of $k$-word matches in biological sequences
}

\author{
November 9, 2018
}

\author{
Sylvain Forêt ${ }^{1}$, Susan R. Wilson ${ }^{1}$, Conrad J. Burden ${ }^{1,2,3}$ \\ ${ }^{1}$ Centre for Bioinformation Science, Mathematical Sciences Institute, The Aus- \\ tralian National University, Canberra ACT 0200, Australia \\ ${ }^{2}$ John Curtin School of Medical Research, The Australian National University, \\ Canberra ACT 0200, Australia \\ ${ }^{3}$ Corresponding author, e-mail: Conrad.Burden@anu.edu.au
}

\begin{abstract}
This study focuses on an alignment-free sequence comparison method: the number of words of length $k$ shared between two sequences, also known as the $D_{2}$ statistic. The advantages of the use of this statistic over alignment-based methods are firstly that it does not assume that homologous segments are contiguous, and secondly that the algorithm is computationally extremely fast, the runtime being proportional to the size of the sequence under scrutiny. Existing applications of the $D_{2}$ statistic include the clustering of related sequences in large EST databases such as the STACK database. Such applications have typically relied on heuristics without any statistical basis. Rigorous statistical characterisations of the distribution of $D_{2}$ have subsequently been undertaken, but have focussed on the distribution's asymptotic behaviour, leaving the distribution of $D_{2}$ uncharacterised for most practical cases. The work presented here bridges these two worlds to give usable approximations of the distribution of $D_{2}$ for ranges of parameters most frequently encountered in the study of biological sequences.
\end{abstract}

\section{Introduction}

The accelerating rate of accumulation of molecular sequences in public databases has triggered the development of a number of sequence comparison algorithms. 
The most popular algorithms, such as FASTA, BLAST or BLAT, rely on sequence alignment, and assume contiguity between homologous segments. This assumption is, however, often broken in molecular sequences, due to events such as transposition, unequal crossing over or alternative splicing. To address this issue, a number of alignment-free sequence comparison methods have been developed. Amongst them, the count of words of length $k$ letters matching between two sequences, also known as the $D_{2}$ statistic, has found some successful applications, due to its simplicity and its speed. The algorithm to calculate the $D_{2}$ statistic between two sequences runs as a linear function of the sequences' lengths, whereas alignmentbased sequence comparison methods typically have a worst case runtime quadratic in the sequences' lengths. The first applications of the $D_{2}$ statistic relied on heuristics to decide whether sequences are significantly similar, but did not have any statistical basis.

A rigorous examination of the distribution of $D_{2}$ led to the characterisation of asymptotic distributions, but the behaviour of $D_{2}$ in practical cases remains unknown. In a previous study, we characterized $D_{2}$ optimal word sizes for a range of sequence sizes. The goal of the present study is to find approximations of the distribution of $D_{2}$ for word sizes close to optimal, and for the sequence sizes most frequently encountered in molecular databases.

\section{Background}

The $D_{2}$ statistic is defined to be the number of exact word matches of length $k$ between sequences $\mathbf{A}=\left(A_{1}, \ldots, A_{m}\right)$ and $\mathbf{B}=\left(B_{1}, \ldots, B_{n}\right)$, with $A_{i}$ and $B_{j}$ belonging to a given alphabet $\mathcal{A}$. For mathematical convenience we will impose periodic boundary conditions on both sequences, that is, the letter in the first position in sequence $\mathbf{A}$ is assumed to follow the letter in the $m$ th position, and the letter in the first position in sequence $\mathbf{B}$ is assumed to follow the letter in the $n$th position. For $k<<m, n$ we do not expect our results to differ significantly from the usual case of free boundary conditions.

Defining the indicator variables $Y_{(i j)}$ for a word match between the $k$-word at position $i$ in $\mathrm{A}$ and the word at position $j$ in $\mathrm{B}$ by

$$
Y_{(i j)}= \begin{cases}1 & \text { if }\left(A_{i}, \ldots, A_{(i+k-1) \bmod m}\right)=\left(B_{j}, \ldots, B_{(j+k-1) \bmod n}\right) \\ 0 & \text { otherwise }\end{cases}
$$

the $D_{2}$ statistic is given by

$$
D_{2}=\sum_{(i, j) \in I} Y_{(i j)}
$$

where $I=\{(i, j) \mid 1 \leq i \leq m, 1 \leq j \leq n\}$. For the case of free boundary conditions the index set is replaced by $I=\{(i, j) \mid 1 \leq i \leq m-k+1,1 \leq j \leq n-k+1\}$. 
We are interested in the distributional properties of $D_{2}$ under the null hypothesis that $\mathbf{A}$ and $\mathbf{B}$ are Bernoulli texts, meaning that each letter, $A_{i}$ or $B_{j}$, is independently and identically (i.i.d.) distributed. Let the probability of occurrence of letter $a \in \mathcal{A}$ be $f_{a}$, and define

$$
p_{t}=\sum_{a \in \mathcal{A}} f_{a}{ }^{t}, \quad t=1,2, \ldots
$$

The mean of $D_{2}$ is then [7]:

$$
E\left(D_{2}\right)=\sum_{(i, j) \in I} E\left(Y_{(i j)}\right)=m n\left(\sum_{a \in \mathcal{A}} f_{a}{ }^{2}\right)^{k}=m n p_{2}{ }^{k} .
$$

An exact value for the variance of $D_{2}$ has recently been given for the case of free boundary conditions in [6]. In Appendix I we derive a similar formula for the variance for the algebraically simpler case of periodic boundary conditions.

From here on, to simplify matters we set $m=n$. Rigorous results exist for the limiting distribution of $D_{2}$ as $n \rightarrow \infty$ in certain regimes. For pairs of Bernoulli texts with non-uniform letter distributions, the limiting distribution is compound Poisson in the regime $k>2 \log _{b} n+$ const. [7], and normal in the regime $k<$ $1 / 2 \log _{b} n+$ const [2]. Here $b=p_{2}{ }^{-1}$.

In earlier numerical analyses [4, we tested the accuracy with which $k$-word matches are able to measure the relatedness of artificially evolved sequences. Calculations of the optimum word size $k$ for a range of sequence lengths $n$, showed that optimum word sizes generally fall between the two parameter regimes for which the asymptotic behaviour of $D_{2}$ is known. Our purpose here is to perform numerical experiments to fill in the gap in the biologically relevant parameter regime between the asymptotically normal and compound Poisson asymptotic behaviours, and to find accurate and practical approximations to the distribution of $D_{2}$ in this parameter region. In particular, we are concerned with accurately reproducing the region of the tail corresponding to classical significance levels $(0.001 \%, 0.01 \%$, $\ldots$ ), both for the distribution of $D_{2}$, and for its extreme value distribution that is used for determining p-values in database searches.

\section{Simulations of the empirical distribution}

The distribution of $D_{2}$ was simulated for a number of combinations of sequence size $n$, word size $k$, alphabets $\mathcal{A}$ and sequence composition $f_{a}$. For nucleic acid sequences, word sizes close to the optimal word size were chosen, based on computation of the optimal word size of $D_{2}$ [4]. We focused on sequence sizes typical of ESTs, whole genome shotgun sequencing trace pairs, CDSs, and mRNAs (100 $\leq n \leq 3200$ bases). For protein amino acid sequences, the optimal word sizes 
and a letter composition equal to the average of the proteins encoded by the human genome where determined using the same method. For protein sequences of length up to $n=400$ the optimum word size was $k=3$, and for longer sequences up to $n=3200$ the optimum word size was $k=4$. The sequence sizes for proteins ranged from small peptides to large proteins (10 to 2560 residues). Sequences were simulated with uniform and non-uniform letter distributions.

For each combination of parameters, $N_{\text {sample }}=10^{6}$ pairs of Bernoulli text sequences were generated. The extreme value distribution was simulated by taking the largest value of 100 comparisons $N_{\text {sample }}$ times. The code for the simulations was written in ANSI C and is available from the author's website [1].

\section{Comparison between empirical and hypoth- esised distributions}

Previous studies of the $D_{2}$ statistic used Kolmogorov-Smirnov tests [3] to compare the empirical distribution of $D_{2}$ with its theoretical asymptotic distributions (normal or compound-Poisson) [7, 2, These studies, however, have been in error for the following reason. Care must be taken when using the Kolmogorov-Smirnov test to pre-specify the parameters of the distribution being compared. If instead, parameters are estimated from the empirical distribution, the p-values obtained will be overestimated (see Appendix II). Given that these earlier studies generally pre-dated the discovery of an analytic formula for the variance of $D_{2}$, they relied on means and variances estimated from empirical samples, and therefore led to overly optimistic claims of agreement between the distribution of the $D_{2}$ statistic for finite length sequences and its theoretical asymptotic limit.

We have repeated Kolmogorov-Smirnov tests of our empirically generated data, standardised with the analytically determined mean and variance of $D_{2}$, against the standard normal distribution. In general, we find p-values to be smaller than those reported in earlier studies. Similar results were obtained using the ShapiroWilk test, which tests for normality but does not require prior knowledge of the mean or variance. More importantly, we find that the information provided by such comparison is rather limited, as the p-value of the Kolmogorov-Smirnov test decreases noticeably with the sample size $N_{\text {sample }}$, since the true distribution of $D_{2}$ for finite sequence length $n$ never exactly matches the hypothesised limiting distribution. We conclude that this type of measure does not give a panacea for how well (or how badly) a given hypothesised distribution will approximate the distribution of $D_{2}$.

Most practical uses of the $D_{2}$ statistic involve the calculation of a p-value resulting from the comparison of two sequences or from the comparison of a query 
sequence to a sequence database. Our approach therefore is to compare a hypothesised distribution with an empirically generated distribution of $D_{2}$ based on a direct comparison of the p-values obtained with these two distributions. If the p-values of a given hypothesised distribution agree well with those of the empirical distribution, this hypothesised distribution could be used to approximate the relevant tail of the real distribution of $D_{2}$.

Suppose we wish to compare a postulated distribution function $F_{\text {hyp }}$ with an empirically generated sample $\left\{x_{1}, \ldots, x_{N_{\text {sample }}}\right\}$. To evaluate how accurately pvalues predicted by $F_{\text {hyp }}$ would approximate those of the true distribution of $D_{2}$, the quantiles

$$
q_{\text {hyp }}=F_{\text {hyp }}^{-1}\left(1-p_{\text {hyp }}\right)
$$

are first calculated for to a number of p-values, $p_{\text {hyp }}$. The frequency, in the simulated data, of the occurrences of $D_{2}$ greater than $q_{\mathrm{hyp}}$ then provides an empirical p-value,

$$
p_{\text {emp }}=\frac{\left|\left\{x_{i}: x_{i} \geq q_{\mathrm{hyp}}\right\}\right|}{N_{\text {sample }}} .
$$

This is compared to $p_{\text {hyp }}$ :

$$
\delta=\left|\log \left(\frac{p_{\text {emp }}}{p_{\text {hyp }}}\right)\right| .
$$

The comparisons focussed on p-values in the range of classical significance levels $\left(p_{\text {hyp }} \in\{0.001 \%, 0.01 \%, \ldots\}\right)$. The theoretical distributions were parameterized using the exact values of $D_{2}$ 's mean and variance. Zero values of $p_{\text {emp }}$ were replaced by $1 / N_{\text {sample. }}$. The hypothesised distributions considered were the normal and gamma distributions. The process is illustrated in Fig. 1

When doing database searches, a query sequence is compared to several sequences, and the p-value of the best score of all these comparisons needs to be estimated. The relevant statistic in this case is the extreme value, that is, the maximum of a number of i.i.d. random variables. In addition to evaluating the tail of the distribution of $D_{2}$ itself, the tail of the empirical extreme value distribution of $D_{2}$ was also compared to those of the the normal and the gamma distributions. These two extreme value distributions belong to the Gumbel family and can be easily computed (see Appendix III).

\section{Results}

\subsection{Approximating the distribution of $D_{2}$}

We first assessed the approximation of the distribution of $D_{2}$ with the normal distribution. Figure 2 shows the results of the comparison of the p-values in the case of nucleic sequences with a uniform letter distribution. Similar results were 


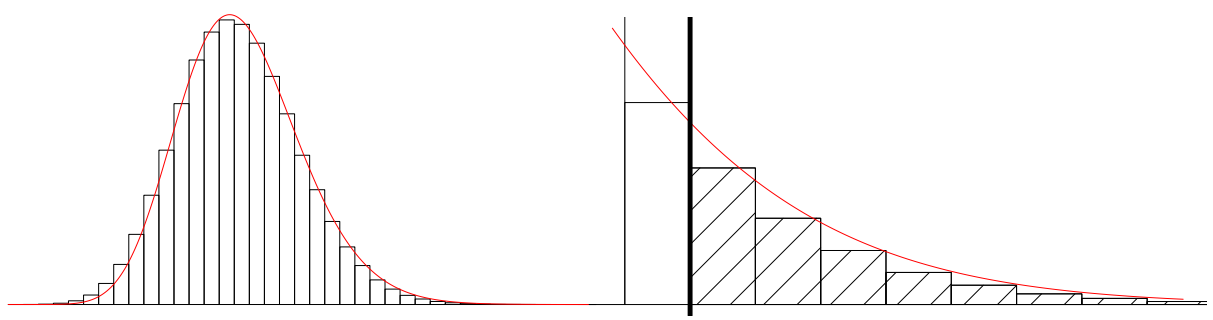

(a)

(b)

Figure 1: Distribution of $D_{2}$ for $n=800, k=7$. The histogram shows the empirical distribution, and the continuous curve is a hypothesised gamma distribution with mean given by Eq. 4 and variance from the calculation in Appendix I. (a) Global view of the distribution. (b) Detail of the right hand tail of the distribution, the vertical bar shows the quantile $q_{\mathrm{hyp}}$, the area under the curve is the corresponding level $p_{\text {hyp }}$, the hatched area is the empirical level $p_{\text {emp }}$.

obtained with non-uniform letter distributions. For sequences 1600 base pairs long or larger, the p-values from the hypothesised normal distribution were very close to the empirical p-values. For smaller sequences and large p-values (up to 1\%), the normal and empirical p-values were of the same order of magnitude. For smaller pvalues, the hypothesised normal distribution greatly overestimated the significance of $D_{2}$. A few other distributions were compared to the simulated distribution of $D_{2}$. The gamma distribution, in particular, approximated the distribution of $D_{2}$ better than the normal distribution did. In this case, the real p-values tended to be overestimated, and the relative difference increased as the p-values decreased (figure 3).

The trends were identical for the amino acid alphabet (figure 4): the normal distribution approximates the p-values relatively well for large sequences and moderate significance levels, but for shorter sequences and further into the tail of the distribution, p-values were strongly overestimated. The gamma distribution generally underestimated the p-values, but was closer to the simulated distribution of $D_{2}$ (figure 5 ).

\subsection{Extreme value distribution}

Figure 6 shows the results of comparison between the extreme value distribution of $D_{2}$, and the extreme values of the gamma and normal distributions in the case of a uniform nucleotide letter distribution for $p_{\text {hyp }}$ in the range $0.1 \%$ to $10 \%$. The extreme value distribution of $D_{2}$ is generally better approximated by the maximum of gamma distributions. Since it was noted in the previous section that the relative difference between the distribution of $D_{2}$ and the normal or the gamma distribution 

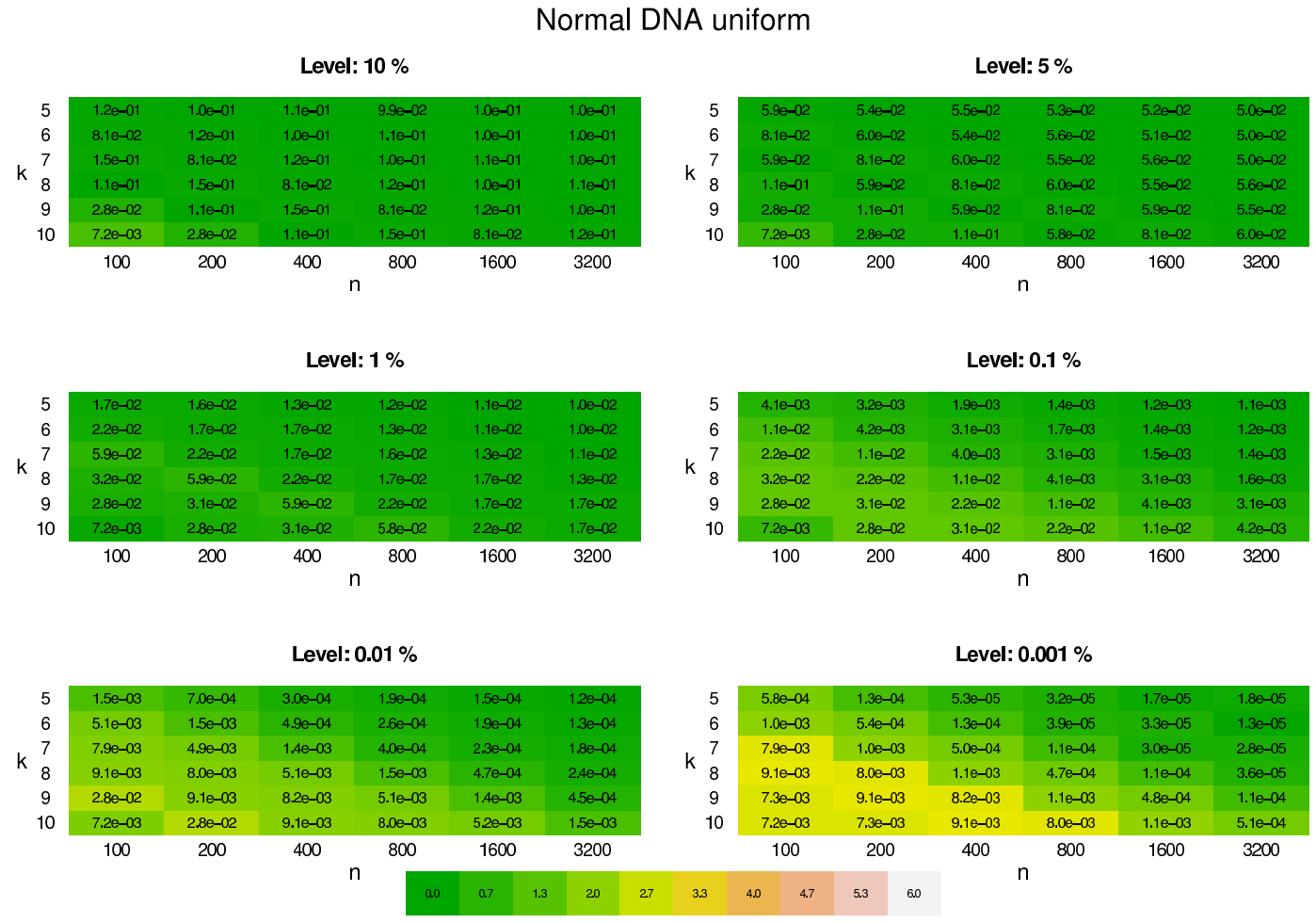

Figure 2: Normal distribution versus empirical distribution of $D_{2}$, DNA alphabet with uniform letter distribution. Each table compares the two distributions at a given level of the hypothesised distribution, for a number of combinations of sequence lengths $n$ and word sizes $k$. The value in each cell corresponds to the empirical level. The colour of each cell reflect the value of $\delta$, as introduced in Eq. 7 . 

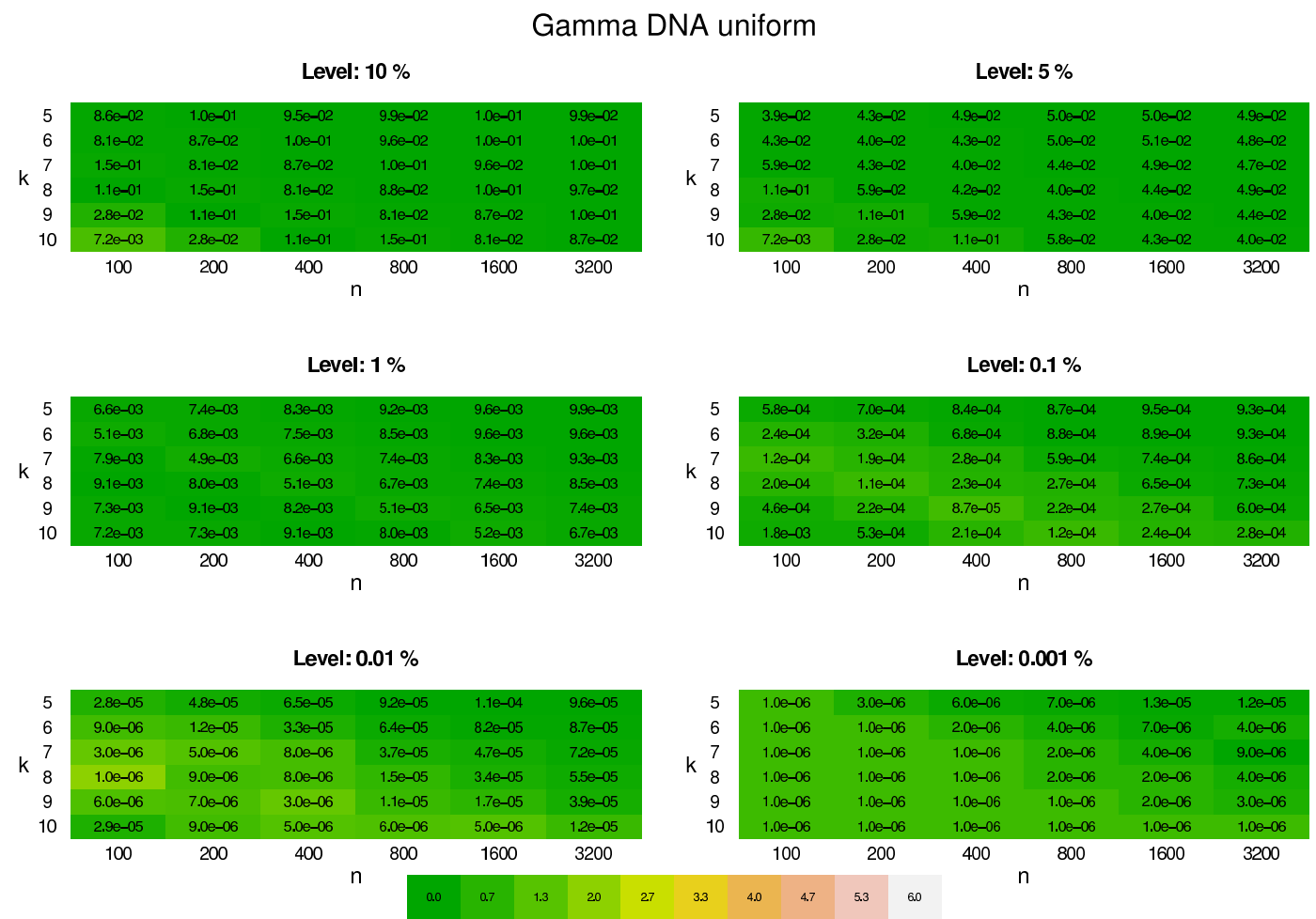

Figure 3: Gamma distribution versus empirical distribution of $D_{2}$, DNA alphabet with uniform letter distribution. See legend of figure 2. 


\section{Normal Protein}

Level: $10 \%$
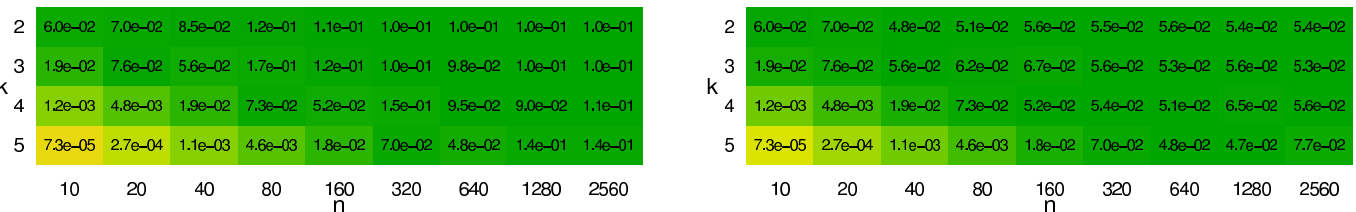

Level: $1 \%$

\section{Level: $0.1 \%$}
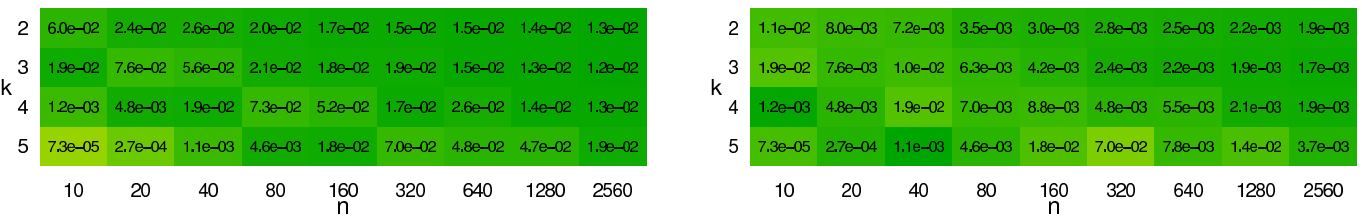

Level: $\mathbf{0 . 0 1} \%$

Level: $0.001 \%$

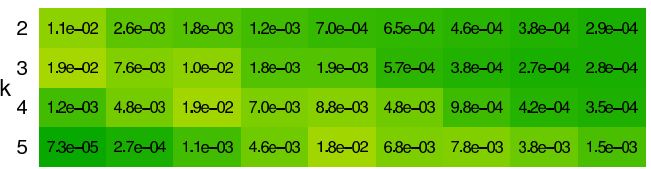

$\begin{array}{llllllllll}2 & 1.9 e-03 & 2.6 e-03 & 4.4 e-04 & 3.9 e-04 & 2.2 e-04 & 1.7 e-04 & 1.1 e-04 & 7.1 e-05 & 4.9 e-05\end{array}$

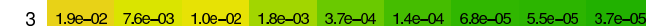

$\begin{array}{lllllllllll}\mathrm{K}_{4} & 1.2 \mathrm{e}-03 & 4.8 \mathrm{e}-03 & 1.9 \mathrm{e}-02 & 7.0 \mathrm{e}-03 & 8.8 \mathrm{e}-03 & 1.2 \mathrm{e}-03 & 4.0 \mathrm{e}-04 & 1.5 \mathrm{e}-04 & 4.2 \mathrm{e}-05\end{array}$

$\begin{array}{lllllllllll}5 & 7.3 \mathrm{e}-05 & 2.7 \mathrm{e}-04 & 1.1 \mathrm{e}-03 & 4.6 \mathrm{e}-03 & 1.8 \mathrm{e}-02 & 6.8 \mathrm{e}-03 & 7.8 \mathrm{e}-03 & 9.6 \mathrm{e}-04 & 2.2 \mathrm{e}-04\end{array}$

$\begin{array}{lllllllll}10 & 20 & 40 & 80 & 160 & 320 & 640 & 1280 & 2560\end{array}$ $\begin{array}{lllllllll}10 & 20 & 40 & 80 & 160 & 320 & 640 & 1280 & 2560\end{array}$

Figure 4: Normal distribution versus empirical distribution of $D_{2}$, amino acid alphabet. See legend of figure 2 . 


\section{Gamma Protein}

Level: $10 \%$

Level: $5 \%$
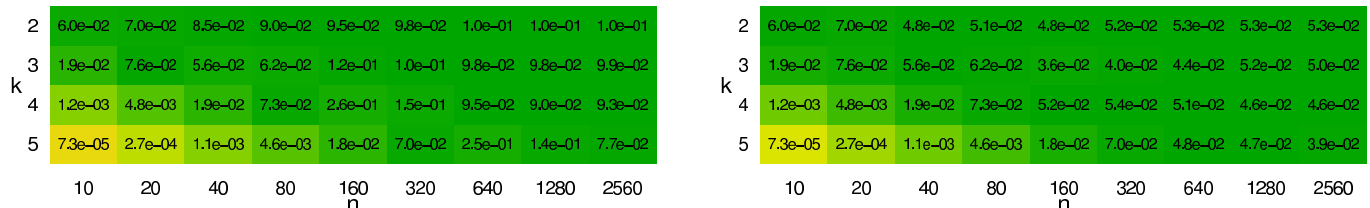

Level: $1 \%$

\section{Level: $0.1 \%$}
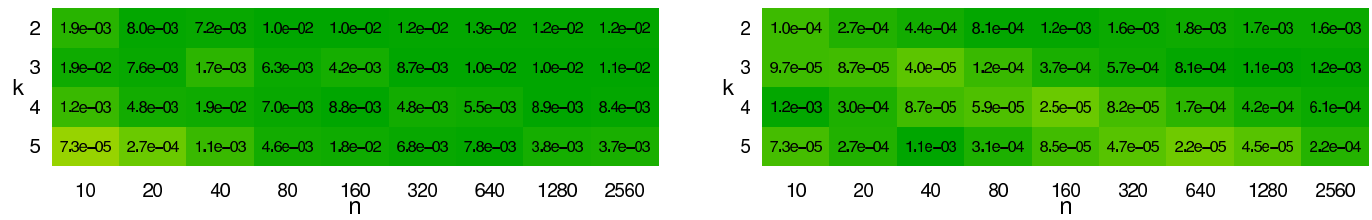

Level: $0.01 \%$

Level: $0.001 \%$
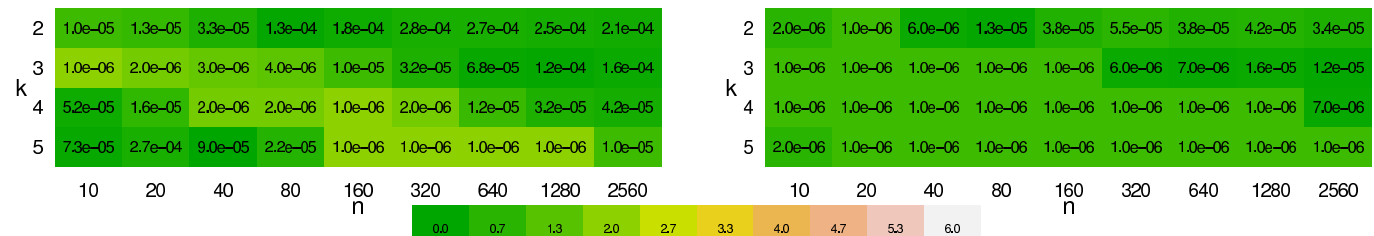

3

k 4

$\begin{array}{lllllllll}100-06 & 1.0 \mathrm{e}-06 & 1.0 \mathrm{e}-06 & 1.0 \mathrm{e}-06 & 1.0 \mathrm{e}-06 & 1.0 \mathrm{e}-06 & 1.0 \mathrm{e}-06 & 1.0 \mathrm{e}-06 & 7.0 \mathrm{e}-06\end{array}$

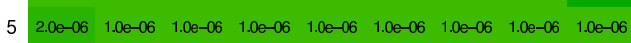
$\begin{array}{lllllllll}10 & 20 & 40 & 80 & 160 & 320 & 640 & 1280 & 2560\end{array}$

Figure 5: Gamma distribution versus empirical distribution of $D_{2}$, amino acid alphabet. See legend of figure 2 . 
increased as the p-values decreased, it is not surprising that the approximations of the extreme value distribution of $D_{2}$ are not as good as the approximations to the distribution of $D_{2}$. The same trends were observed for nucleic sequences of non-uniform letters and amino acid sequences.

\section{Discussion and Conclusions}

This study introduces practical approximations to the distribution of the $D_{2}$ statistic and to the extreme value distribution of $D_{2}$. For sequences of intermediate length (around 800 base pairs, close to the average size of ESTs and sequencing traces) and for p-values between $5 \%$ and $0.1 \%$, the Gamma distribution closely approximates the distribution of $D_{2}$. The Gamma distribution not only outperforms the normal distribution, but unlike the latter, it slightly overestimates the p-values, and thus would result in fewer false positives.

All the approximations presented here deteriorate as one moves further to the right hand of the tail (for smaller p-values). This is not, however, a major problem for any practical use of these approximations, where very small p-values would just have an indicative value.

Finally, our results show, that for longer sequences, such as genome assembly contigs, the normal approximation itself would be appropriate, even for very small p-values.

\section{Appendices}

\section{Calculation of $\operatorname{Var} D_{2}$}

Using Eq. 2, the variance of $D_{2}$ is

$$
\operatorname{Var}\left(D_{2}\right)=\operatorname{Var}\left(\sum_{(i, j) \in I} Y_{(i j)}\right)=\sum_{(i, j) \in I} \operatorname{Var}\left(Y_{(i j)}\right)+\sum_{(i, j) \neq\left(i^{\prime}, j^{\prime}\right)} \operatorname{Cov}\left(Y_{(i j)}, Y_{\left(i^{\prime} j^{\prime}\right)}\right)
$$

To simplfy the notation from here on we set $u=(i, j), v=\left(i^{\prime}, j^{\prime}\right)$. The first term in Eq. 8 depends only on

$$
\operatorname{Var}\left(Y_{u}\right)=E\left(Y_{u}^{2}\right)-\left(E\left(Y_{u}\right)\right)^{2}=E\left(Y_{u}\right)-\left(E\left(Y_{u}\right)\right)^{2}={p_{2}}^{k}-p_{2}{ }^{2 k},
$$

where $p_{t}$ is defined in Eq. 3. Thus

$$
\sum_{u \in I} \operatorname{Var}\left(Y_{u}\right)=m n\left(p_{2}{ }^{k}-p_{2}{ }^{2 k}\right) .
$$


Gumbel (Normal) DNA uniform

Level: $10 \%$

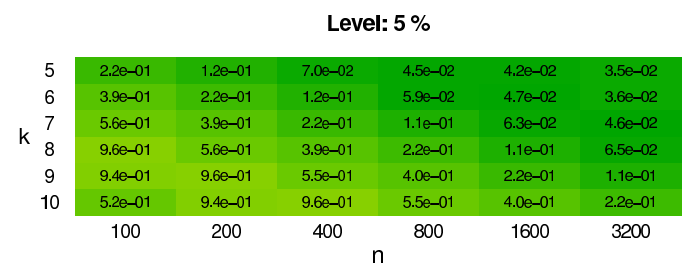

Level: $1 \%$

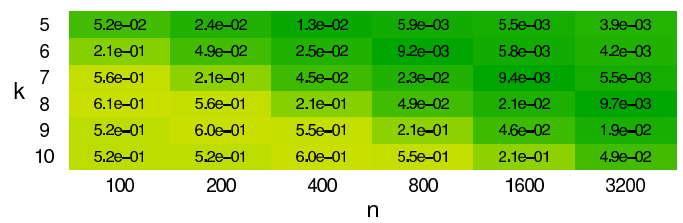

Level: $0.1 \%$
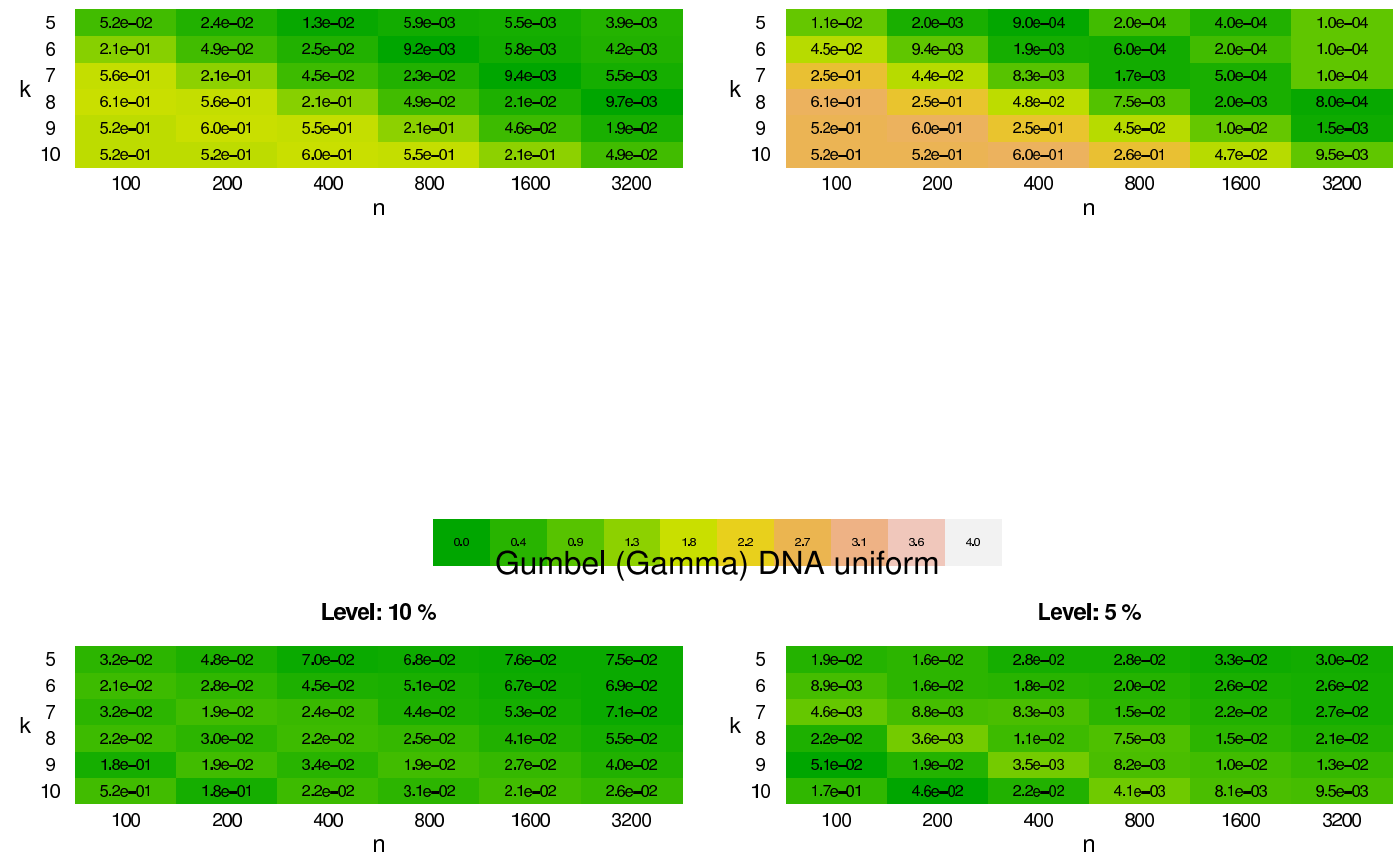

Level: $1 \%$

Level: $0.1 \%$
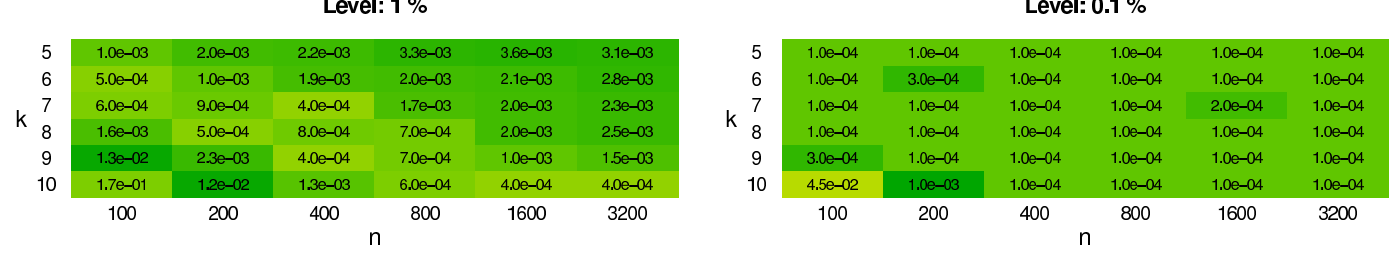

\begin{tabular}{|l|l|l|l|l|l|l|l|l|l|}
\hline 0.0 & 0.4 & 0.9 & 1.3 & 1.8 & 2.2 & 2.7 & 3.1 & 3.6 & 4.0
\end{tabular}

Figure 6: Extreme value of Normal and Gamma versus empirical extreme value of $D_{2}$, DNA alphabet with uniform letter distribution. See legend of figure 2 . 
To calculate the covariances in the second term of Eq. 8, it is convenient to use the notation and terminology of [8], Chapter 11. Let $J_{u}=\left\{v=\left(i^{\prime}, j^{\prime}\right):\left|i^{\prime}-i\right|<\right.$ $k$ or $\left.\left|j^{\prime}-j\right|<k\right\}$ be the dependency neighbourhood of $Y_{u}$. It can be decomposed into two parts, accordion and crabgrass, $J_{u}=J_{u}^{a} \cup J_{u}^{c}$, where

$$
\left.J_{u}^{a}=\left\{v=\left(i^{\prime}, j^{\prime}\right) \in J_{u}:\left|i^{\prime}-i\right|<k \text { and }\left|j^{\prime}-j\right|<k\right\}\right\} \quad \text { and } \quad J_{u}^{c}=J_{u} \backslash J_{u}^{a} .
$$

We compute the cross covariances, $\operatorname{Cov}\left(Y_{u}, Y_{v}\right)$, by looking at the following cases. Case 1: $v \notin J_{u}$. In this case, $Y_{u}$ and $Y_{v}$ are independent and hence $\operatorname{Cov}\left(Y_{u}, Y_{v}\right)=$ 0 .

Case 2: $v \in J_{u}^{c}$. Let $u=(i, j)$ and $v \in J_{u}^{c}$. Consider first the subcase $v=\left(i+t, j^{\prime}\right)$, where $\left|j-j^{\prime}\right| \geq k$ and $0 \leq t \leq k-1$. Then

$$
\begin{aligned}
E\left(Y_{u} Y_{v}\right) & =\operatorname{Pr}\left(Y_{u}=1, Y_{v}=1\right) \\
& =\sum_{\left(a_{1}, \ldots, a_{k+t}\right) \in \mathcal{A}}\left(f_{a_{1}} \ldots f_{a_{k+t}}\right)\left(f_{a_{1}} \ldots f_{a_{k}}\right)\left(f_{a_{1+t}} \ldots f_{a_{k+t}}\right) \\
& =\left(\sum_{a \in \mathcal{A}} f_{a}{ }^{2}\right)^{2 t}\left(\sum_{a \in \mathcal{A}} f_{a}{ }^{3}\right)^{k-t} \\
& =p_{2}{ }^{2 t} p_{3}{ }^{k-t},
\end{aligned}
$$

where we have used the fact that word matches occur simultaneously at $u$ and $v$ if and only if the first $k$ letters of $\left(A_{i}, \ldots, A_{i+k+t-1}\right)$ are repeated at $\left(B_{j}, \ldots, B_{j+k-1}\right)$ and the final $k$ letters are repeated at $\left(B_{j^{\prime}}, \ldots, B_{j^{\prime}+k-1}\right)$. This gives

$$
\operatorname{Cov}\left(Y_{u}, Y_{v}\right)=E\left(Y_{u} Y_{v}\right)-E\left(Y_{u}\right) E\left(Y_{v}\right)=p_{2}{ }^{2 t} p_{3}{ }^{k-t}-p_{2}{ }^{2 k} .
$$

Extending the argument to all $-k+1 \leq t \leq k-1$ gives

$$
\operatorname{Cov}\left(Y_{u}, Y_{v}\right)={p_{2}}^{2|t|} p_{3}{ }^{k-|t|}-p_{2}{ }^{2 k} \text {. }
$$

By symmetry of the covariance function, the same result applies to the sub-case $v=\left(i^{\prime}, j+t\right)$ where $\left|i-i^{\prime}\right| \geq k$ and $|t| \leq k-1$.

The crabgrass contribution to the sum over covariance terms in Eq. 8 is then

$$
\begin{aligned}
& \sum_{u} \sum_{v \in J_{u}^{c}} \operatorname{Cov}\left(Y_{u}, Y_{v}\right) \\
& =\sum_{u}\left(\sum_{\left\{j^{\prime}:\left|j^{\prime}-j\right| \geq k\right\}}+\sum_{\left\{i^{\prime}:\left|i^{\prime}-i\right| \geq k\right\}}\right) \sum_{t=-k+1}^{k-1}\left(p_{2}{ }^{2|t|} p_{3}{ }^{k-|t|}-p_{2}{ }^{2 k}\right) \\
& =m n(m+n-4 k+2)\left[p_{3}{ }^{k}+2 \sum_{t=1}^{k-1} p_{2}{ }^{2 t} p_{3}{ }^{k-t}-(2 k-1) p_{2}{ }^{2 k}\right] \\
& =m n(m+n-4 k+2)\left[p_{3}{ }^{k}+2{p_{2}}^{2} p_{3} \frac{p_{3}{ }^{k-1}-p_{2}{ }^{2(k-1)}}{p_{3}-p_{2}{ }^{2}}-(2 k-1) p_{2}{ }^{2 k}\right]
\end{aligned}
$$




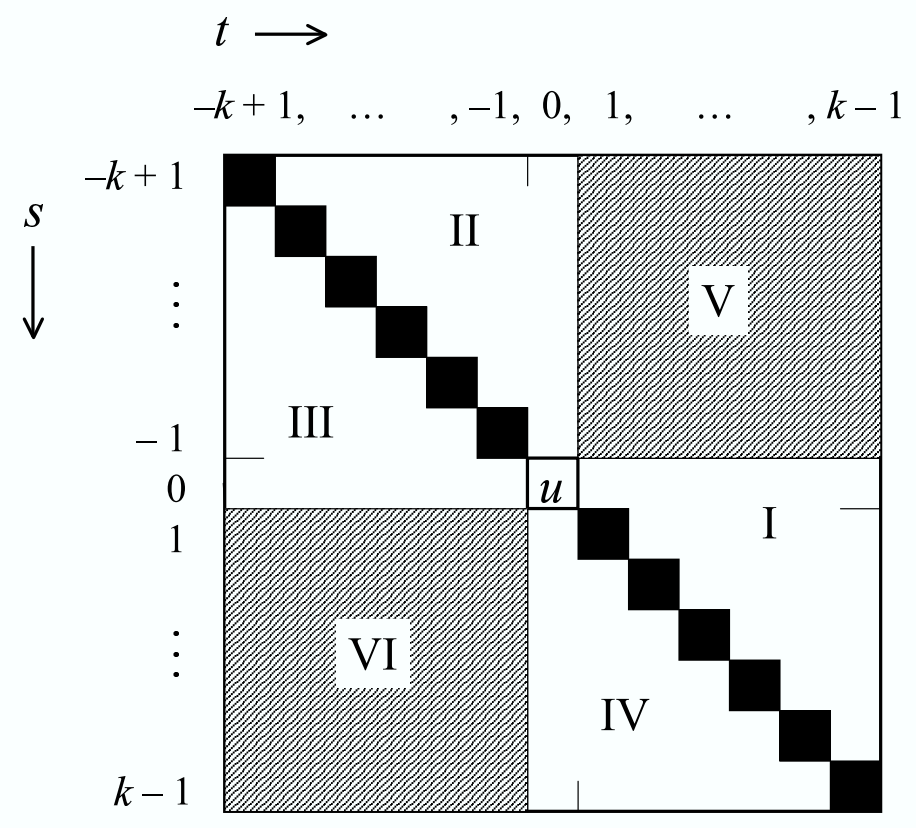

Figure 7: The main diagonal of $J_{u}^{a}$ referred to in Case 3 (black squares), and the sub-regions I to VI referred to in Cases 4 and 5.

Case 3: $v$ is on the main diagonal of $J_{u}^{a}$. That is, $v=(i+t, j+t)$, where $-k<t<k$ and $t \neq 0$ (see Fig. 7). In this case,

$$
\begin{aligned}
E\left(Y_{u} Y_{v}\right) & =\operatorname{Pr}\left(Y_{u}=1, Y_{v}=1\right) \\
& =\operatorname{Pr}(\text { a specific }(k+|t|) \text {-word match at the }(i, j) \text { position }) \\
& =\sum_{\left(a_{1}, \ldots, a_{k+|t|}\right) \in \mathcal{A}^{k+|t|}} f_{a_{1}}^{2} \times \ldots \times f_{a_{k+|t|}}^{2} \\
& =p_{2}{ }^{k+|t|}
\end{aligned}
$$

and

$$
\operatorname{Cov}\left(Y_{u}, Y_{v}\right)=E\left(Y_{u} Y_{v}\right)-E\left(Y_{u}\right) E\left(Y_{v}\right)={p_{2}}^{k+|t|}-p_{2}{ }^{2 k}
$$

The contribution to the sum over covariance terms in Eq. 8 from Case 3 is then

$$
\begin{aligned}
& \sum_{u} \sum_{v \in \text { main diagonal, } v \neq u} \operatorname{Cov}\left(Y_{u}, Y_{v}\right) \\
& =2 m n \sum_{t=1}^{k-1}\left(p_{2}{ }^{t+k}-{p_{2}}^{2 k}\right) \\
& =2 m n\left[p_{2}{ }^{k+1} \frac{1-p_{2}{ }^{k-1}}{1-p_{2}}-(k-1) p_{2}{ }^{2 k}\right] .
\end{aligned}
$$

Case 4: $v \in$ one of the subregions I, II, III or IV of $J_{u}^{a}$ in Fig. 7. That is, $v=(i+t, j+s)$, where 
I: $0 \leq s<t \leq k-1$

II: $-k+1 \leq s<t \leq 0$

III: $-k+1 \leq t<s \leq 0$ or

IV: $0 \leq t<s \leq k-1$.

Consider subregion I first. The word matches corresponding to the event " $Y_{u}=$ $1, Y_{v}=1 "$ are illustrated in Fig. 8. For such a situation to occur, the $t+s$ letters $a_{1}, \ldots, a_{s}, b_{1}, \ldots, b_{s}$ and $c_{1}, \ldots, c_{t-s}$ can be specified independently, and the remaining $2 k$ letters within the four words must be repeats of $c_{1}, \ldots, c_{t-s}$ as shown. The sequence $c_{1}, \ldots, c_{t-s}$ is repeated $\nu=\lfloor(k-s) /(t-s)\rfloor$ complete times in sequence $B$ and $\nu+1$ complete times in sequence $A$, where \lfloor\rfloor indicates the integer part. At the right hand end of these repeats, the sequence $c_{1}, \ldots, c_{\rho}$ occurs once in Sequence $A$ and once in Sequence $B$, where $\rho=(k-s) \bmod (t-s)$.

Then

$$
\begin{aligned}
& E\left(Y_{u} Y_{v}\right)=\operatorname{Pr}\left(Y_{u}=1, Y_{v}=1\right) \\
& =\sum_{\left(a_{1}, \ldots, a_{s}, b_{1}, \ldots, b_{s}, c_{1}, \ldots, c_{t-s}\right) \in \mathcal{A}^{t+s}} f_{a_{1}}{ }^{2} \ldots f_{a_{s}}{ }^{2} f_{c_{1}}{ }^{2 \nu+3} \ldots f_{c_{\rho}}{ }^{2 \nu+3} \times \\
& f_{c_{\rho+1}}{ }^{2 \nu+1} \ldots f_{c_{t-s}}{ }^{2 \nu+1} f_{b_{1}}{ }^{2} \ldots f_{b_{s}}{ }^{2} \\
& =\left(\sum_{a \in \mathcal{A}} f_{a}{ }^{2}\right)^{s}\left(\sum_{c \in \mathcal{A}} f_{c}{ }^{2 \nu+3}\right)^{\rho}\left(\sum_{c \in \mathcal{A}} f_{c}{ }^{2 \nu+1}\right)^{t-s-\rho}\left(\sum_{b \in \mathcal{A}} f_{a}{ }^{2}\right)^{s} \\
& =p_{2}{ }^{2 s} p_{2 \nu+3}{ }^{\rho} p_{2 \nu+1}{ }^{t-s-\rho},
\end{aligned}
$$

and

$$
\operatorname{Cov}\left(Y_{u}, Y_{v}\right)=E\left(Y_{u} Y_{v}\right)-E\left(Y_{u}\right) E\left(Y_{v}\right)={p_{2}}^{2 s} p_{2 \nu+3}{ }^{\rho} p_{2 \nu+1}{ }^{t-s-\rho}-p_{2}{ }^{2 k} .
$$

It is straightforward to check that similar results apply to subregions II, III and IV, giving the contribution to the sum over covariances in Eq. 8 from Case 4 as

$$
\sum_{u} \sum_{v \in R} \operatorname{Cov}\left(Y_{u}, Y_{v}\right)=4 n m \sum_{t=1}^{k-1} \sum_{s=0}^{t-1}\left(p_{2}^{2 s} p_{2 \nu+3}{ }^{\rho} p_{2 \nu+1}{ }^{t-s-\rho}-p_{2}^{2 k}\right),
$$

where $R=\mathrm{I} \cup \mathrm{II} \cup \mathrm{III} \cup \mathrm{IV}$ is the union of the four subregions of Case 4 and

$$
\nu=\left\lfloor\frac{k-s}{t-s}\right\rfloor, \quad \rho=(k-s) \bmod (t-s) .
$$

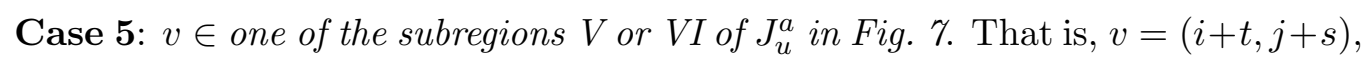
where 


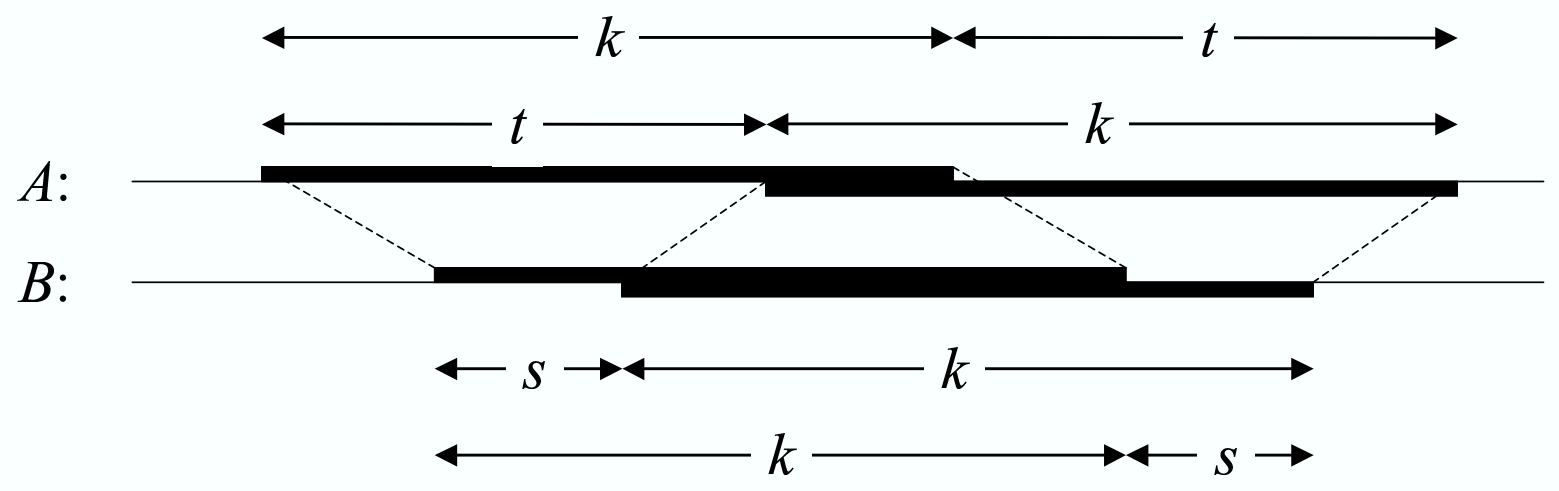

\begin{tabular}{c|c|c|c|c|}
\hline$: \quad a_{1}, \ldots, a_{s}$ & $c_{1}, \ldots, c_{t-s}$ & $c_{1}, \ldots, c_{t-s}$ & $\ldots \ldots$ & $b_{1}, \ldots, b_{s}$ \\
\hline
\end{tabular}

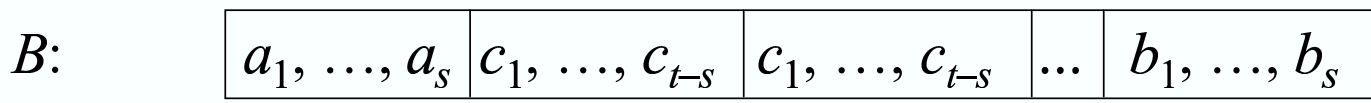

Figure 8: Word match configuration corresponding to Case 4(I). If the letters $a_{1}, \ldots, a_{s}, b_{1}, \ldots, b_{s}$ and $c_{1}, \ldots, c_{t-s}$ are specified, the remaining letters within the four words must be repeats of $c_{1}, \ldots, c_{t-s}$ as shown, the final repeat being truncated at the same point in both Sequence $A$ and Sequence $B$. 


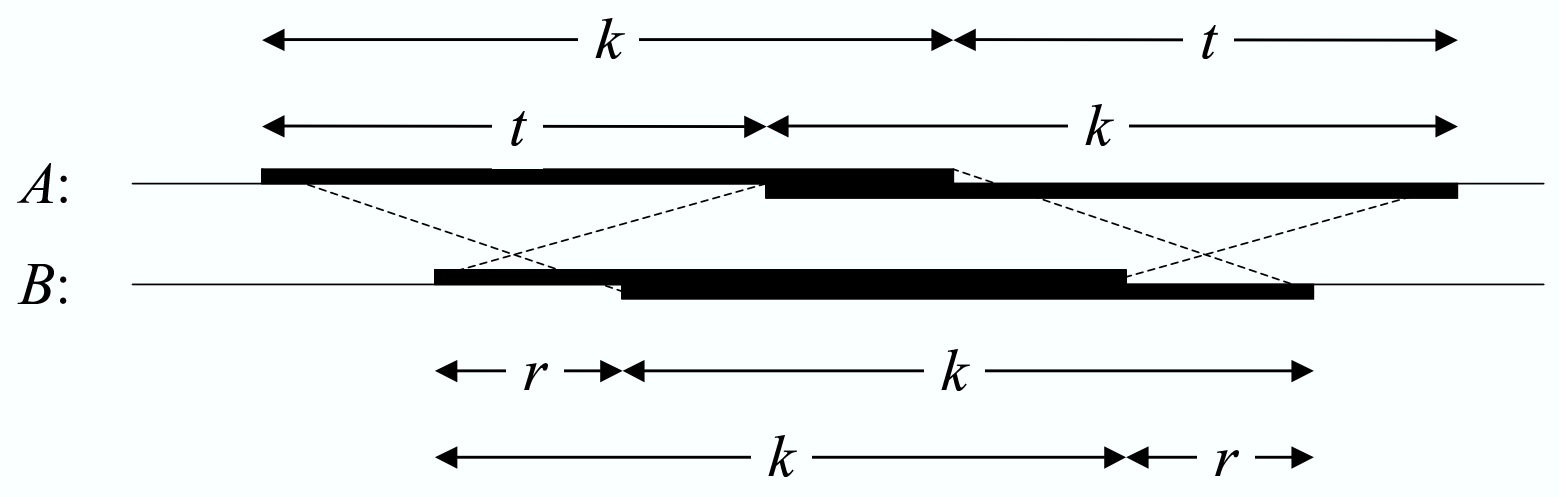

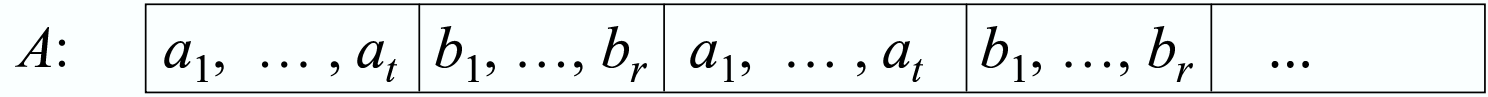

$B: \quad$\begin{tabular}{l|l|l|l|l|}
\hline$b_{1}, \ldots, b_{r}$ & $a_{1}, \ldots, a_{t}$ & $b_{1}, \ldots, b_{r}$ & $\ldots$ \\
\hline
\end{tabular}

Figure 9: Word match configuration corresponding to Case 5(V). If the letters $a_{1}, \ldots, a_{t}, b_{1}, \ldots, b_{r}$ are specified, the remaining letters within the four words must be repeats of $a_{1}, \ldots, b_{r}$ in Sequence $\mathrm{A}$ and $b_{1}, \ldots, a_{t}$ in sequence B, the final repeat being truncated at the $(k+t)$ th or $(k+r)$ th position respectively. 


\section{$\mathrm{V}: 1 \leq t \leq k-1,-k+1 \leq s \leq-1 ;$}

$\mathrm{VI}: 1 \leq s \leq k-1,-k+1 \leq t \leq-1$.

Consider subregion $\mathrm{V}$ first. The word matches corresponding to the event " $Y_{u}=1, Y_{v}=1$ " are illustrated in Fig. 9. Set $r=-s$. For the event to occur, the affected block of length $t+k$ in Sequence A must consist of repeats of a sequence $\left(a_{1}, \ldots, a_{t}, b_{1}, \ldots, b_{r}\right)$ where $a_{1}, \ldots, a_{t}$ and $b_{1}, \ldots, b_{r}$ are independently specified letters. The final repeat is truncated at the $(k+t)$ th letter. The affected block in sequence $\mathrm{B}$ must consist of repeats of the sequence $\left(b_{1}, \ldots, b_{r}, a_{1}, \ldots, a_{t}\right)$, the final repeat being truncated at the $(k+r)$ th letter.

Let $l_{i}, i=1, \ldots, t$ be the total number of times the letter $a_{i}$ occurs and $m_{j}$, $j=1, \ldots, r$ be the total number of times the letter $b_{j}$ occurs in the two blocks in Fig. 9, By noting that the stretches of length $k$ not including the first $t$ letters of the A-block or not including the first $r$ letters of the B-block each contain $\lfloor k /(r+t)\rfloor$ complete repeats of all $s+t$ independent letters plus a final $k \bmod (r+t)$ remaining letters at the right hand end, we arrive at

$$
\begin{gathered}
l_{i}=1+2 \eta+\left\{\begin{array}{ll}
1 & \text { if } i \leq \zeta \\
0 & \text { otherwise }
\end{array}\right\}+\left\{\begin{array}{ll}
1 & \text { if } i \leq \zeta-r \\
0 & \text { otherwise }
\end{array}\right\} \\
m_{j}=1+2 \eta+\left\{\begin{array}{ll}
1 & \text { if } j \leq \zeta \\
0 & \text { otherwise }
\end{array}\right\}+\left\{\begin{array}{ll}
1 & \text { if } j \leq \zeta-t \\
0 & \text { otherwise }
\end{array}\right\},
\end{gathered}
$$

where

$$
\eta=\left\lfloor\frac{k}{r+t}\right\rfloor, \quad \zeta=k \bmod (r+t) .
$$

Then

$$
\begin{aligned}
E & \left(Y_{u} Y_{v}\right)=\operatorname{Pr}\left(Y_{u}=1, Y_{v}=1\right) \\
& =\sum_{\left(a_{1}, \ldots, a_{t}, b_{1}, \ldots, b_{r}\right) \in \mathcal{A}^{t+r}} f_{a_{1}}{ }^{l_{1}} \ldots f_{a_{t}}{ }^{l_{t}} f_{b_{1}}{ }^{m_{1}} \ldots b_{r}{ }^{m_{r}} \\
& =\left(\sum_{a \in \mathcal{A}} f_{a}{ }^{l_{1}}\right) \ldots\left(\sum_{a \in \mathcal{A}} f_{a}^{l_{t}}\right)\left(\sum_{b \in \mathcal{A}} f_{b}{ }^{m_{1}}\right) \ldots\left(\sum_{b \in \mathcal{A}} f_{b}{ }^{m_{r}}\right) \\
& =\left(\prod_{i=1}^{t} p_{l_{i}}\right)\left(\prod_{j=1}^{r} p_{m_{j}}\right),
\end{aligned}
$$

and

$$
\operatorname{Cov}\left(Y_{u}, Y_{v}\right)=E\left(Y_{u} Y_{v}\right)-E\left(Y_{u}\right) E\left(Y_{v}\right)=\left(\prod_{i=1}^{t} p_{l_{i}}\right)\left(\prod_{j=1}^{r} p_{m_{j}}\right)-p_{2}{ }^{2 k}
$$


A similar result holds for subregion VI. The contribution to the sum over covariances in Eq. 8 from Case 5 is then

$$
\sum_{u} \sum_{v \in S} \operatorname{Cov}\left(Y_{u}, Y_{v}\right)=2 n m \sum_{r, t=1}^{k-1}\left[\left(\prod_{i=1}^{t} p_{l_{i}}\right)\left(\prod_{j=1}^{r} p_{m_{j}}\right)-p_{2}^{2 k}\right],
$$

where $S=\mathrm{V} \cup \mathrm{VI}$ is the union of the two subregions of Case 5 and $l_{i}$ and $m_{j}$ are given by Eq. 22,

Finally, by Eq. 8, the variance of $D_{2}$ is given by the sum of the right hand sides of Eqs. 10, 14, 17, 20 and 26.

\section{Consequences of failing to pre-specify parameters in the Kolmogorov-Smirnov test}

Given a random sample of observations $X_{1}, X_{2}, \ldots, X_{N_{\text {sample }}}$, the KolmogorovSmirnov test 3 gives p-values for the null hypothesis that the observations are associated with pre-specified distribution function $F_{\text {hyp }}$. The two-sided version of the test considered here uses as a test statistic $\sup _{i}\left|F_{\text {hyp }}\left(X_{i}\right)-S\left(X_{i}\right)\right|$, where $S$ is the empirical cumulative distribution function based on the observations. Under the null hypothesis the p-values obtained are uniformly distributed on the interval $[0,1]$.

Importantly, if the hypothesised distribution $F_{\text {hyp }}$ is not fully pre-specified, but relies on estimates from the sample, the reported p-values will not be uniformly distributed under the null hypothesis. To illustrate this, we have generated a set of 10,000 independent samples of $N_{\text {sample }}=2500$ random numbers from a standard normal distribution, and applied the two-sided Kolmogorov-Smirnov test to each sample using the R function ks.test. Histograms of the p-values obtained are shown in Fig. 10. In the first plot each sample was tested against the standard normal $N(0,1)$, whereas in the second plot each sample was tested against a normal distribution whose mean and variance was estimated from the sample. We see that in this situation, where the null hypothesis is true, but the Kolmogorov-Smirnov test is applied incorrectly, p-values are skewed heavily towards 1.

In a second test to see whether incorrect use of the Kolmogorov-Smirnov test can lead to an overly optimistic indication of agreement with a hypothesised distribution, we generated a set of 10,000 independent samples of $N_{\text {sample }}=2500$ random numbers from a Gamma distribution with mean 10 and variance 1 . This distribution is close to, but not identical with, the normal distribution $N(10,1)$. The third and fourth histograms in Fig. 10 are of p-values obtained from application of the Kolmogorov-Smirnov test against a pre-specified $N(10,1)$, and against a normal distribution with mean and variance estimated from the sample respectively. The third plot is an indication the distribution of p-values that will result if the Kolmogorov-Smirnov test for normality is applied correctly to this non-normal 

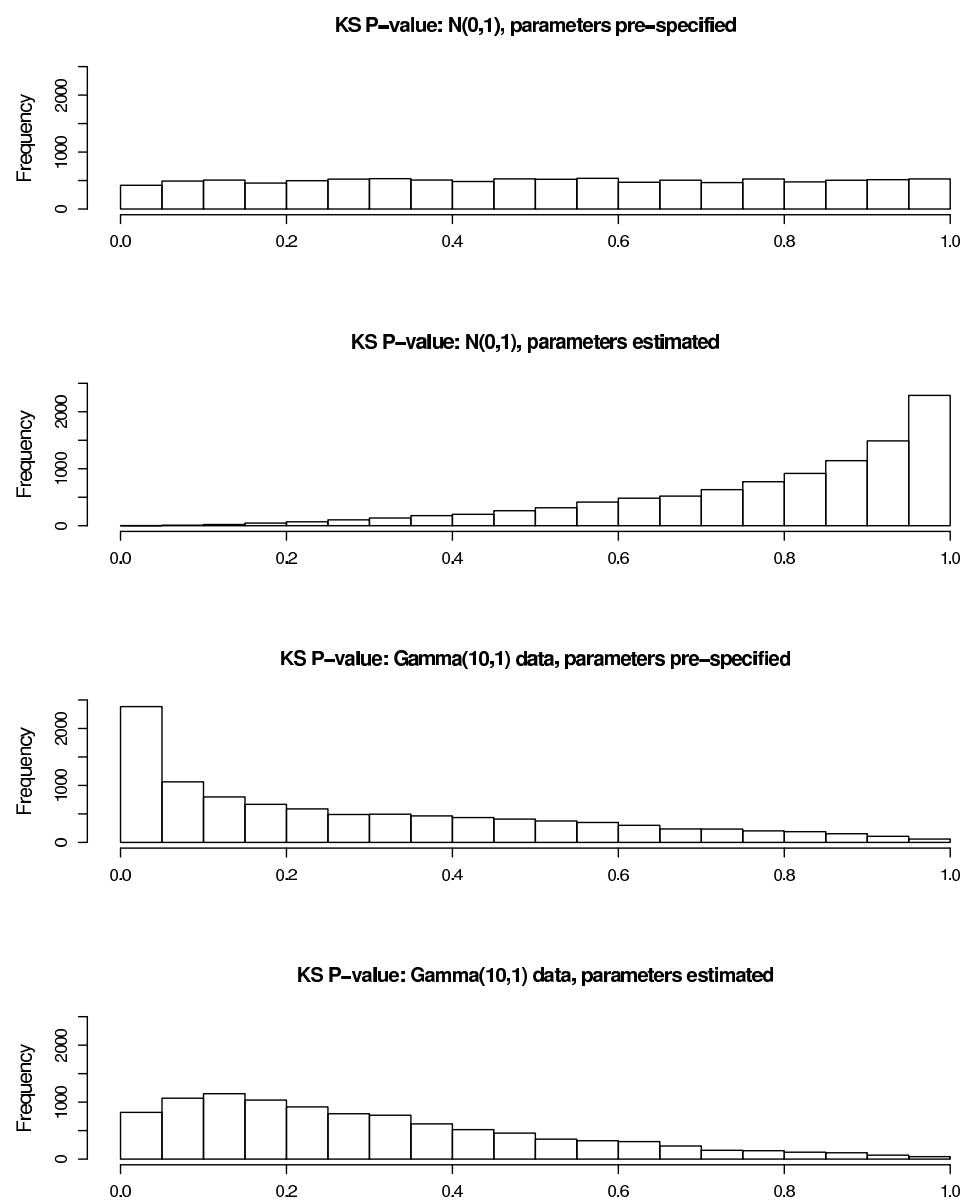

Figure 10: Histograms of p-values obtained from the Kolmogorov-Smirnov test applied to artificially generated data tested against a normal distribution. From top to bottom the plots are (i) standard normal data, parameters of the hypothesised distribution pre-specified; (ii) standard normal data, parameters of the hypothesised distribution estimated from the data; (iii) gamma distributed data with mean 10 and variance 1, parameters of the hypothesised distribution pre-specified; and (iv) gamma distributed data with mean 10 and variance 1, parameters of the hypothesised distribution estimated from the data. 
data. Again we see that p-values are overestimated in the fourth plot when the test is used incorrectly.

\section{Limiting distribution of the maximum of $N$ i.i.d. random variables}

We are interested in the limiting distribution for $N$ large of the random variable

$$
X_{\max }=\max _{i} X_{i},
$$

where $X_{i}, i=1, \ldots, N$ are i.i.d. random variables with common density function $f_{X}$ and distribution function

$$
F_{X}(x)=\int_{-\infty}^{x} f_{X}(\xi) d \xi
$$

The general theory of extreme value distributions is given in the book by Gumbel [5], Chapter 5.2. For distributions of "type I", which includes the normal and gamma distributions, the distribution function of $X_{\max }$, namely $\left(F_{X}(x)\right)^{N}$, asymptotes to the double exponential distribution function

$$
G(x)=\exp \left(-e^{-y}\right),
$$

where the reduced largest value is defined as

$$
y=\alpha_{N}\left(x-u_{N}\right) .
$$

Here $u_{N}$, called the characteristic largest value, is determined by the condition that in $N$ observations of $X$, the expected number of values greater than or equal to $u_{N}$ is unity. It is the solution to the equation

$$
F_{X}\left(u_{N}\right)=1-\frac{1}{N},
$$

and for the case of the normal and gamma distribution is easily found using the $\mathrm{R}$ function qnorm() and qgamma() respectively. The parameter $\alpha_{N}$ is called the extremal intensity function and is given by

$$
\alpha_{N}=N f_{X}\left(u_{N}\right) .
$$

\section{Acknowledgement}

We thank Professors Alan Welsh and Chris Field for inspiring discussions. This work was funded in part by ARC Discovery Grant DP0559260. 


\section{References}

[1] http://dayhoff.anu.edu.au/ sf/k_words.

[2] C. J. Burden, M. R. Kantorovitz, and S. R. Wilson. Approximate word matches between two random sequences. Annals of Applied Probability, 18(1):1-21, 2006.

[3] W.J. Conover. Practical Nonparametric Statistics. John Wiley and Sons, 1999.

[4] S. Forêt, M. R. Kantorovitz, and C. J. Burden. Asymptotic behaviour and optimal word size for exact and approximate word matches between random sequences. BMC Bioinformatics, 7 Suppl 5:S21, 2006.

[5] E. J. Gumbel. Statistics of Extremes. Columbia University Press, New York, 1958.

[6] M. R. Kantorovitz, G. E. Robinson, and S. Sinha. A statistical method for alignment-free comparison of regulatory sequences. Bioinformatics, 23(13):i249-55, 2007.

[7] R. A. Lippert, H. Huang, and M. S. Waterman. Distributional regimes for the number of k-word matches between two random sequences. Proc Natl Acad Sci U S A, 99(22):13980-9, 2002.

[8] M. S. Waterman. Introduction to Computational Biology. Chapman and Hall, 1995.

\section{Vitae}

\section{Sylvain Forêt}

Sylvain Forêt graduated in Invertebrates Physiology and Computer Science at the INAP-G and Université Pierre et Marie Currie in Paris, France in 1998. He received his $\mathrm{PhD}$ in molecular biology and biochemistry from the Australian National University in Canberra, Australia in 2007.

\section{Susan R. Wilson}

Susan Wilson obtained her B.Sc. from the University of Sydney in 1968, and her Ph.D. from the Australian National University in 1972. She is an elected member of the International Statistical Institute, elected Fellow of the American Statistical Association and of the Institute of Mathematical Statistics. She has been President, International Biometric Society. 


\section{Conrad J. Burden}

Conrad J. Burden received his B.Sc. in applied mathematics from the University of Queensland in 1978, his Ph.D. in theoretical physics from the Australian National University in 1983, and is a Fellow of the Australian Institute of Physics. For the first 16 years of his academic career his research interests centred on subatomic particle physics and quantum field theory. After a brief sojourn in the IT industry he made the transition to bioinformation science in 2003. He is currently a Fellow in the Centre for Bioinformation Science at the Australian National University where his research interests include modelling of oligonucleotide microarrays, alignment free sequence comparison methods, gene regulation and protein structure. 